\title{
Nanometre-Scale Methods
}

The Europhysics Industrial Workshop on Nanometre-Scale Methods in Technology and Industry (Davos, 18-21 August 1991) offered an unique opportunity for workers from academic and industrial centres to compare a broad range of $\mathrm{nm}$-scale techniques for both imaging/measurement and manufacturing/manipulation applications. In spite of the interest in the newer local probe techniques, the organizers - J.K. Gimzewski (IBM Rüschlikon) and M.E. Welland (Cambridge) - were able to ensure that the 60 or so participants covered the equally significant, but more conventional, non-local probe methods.

H. Rohrer (IBM Rüschlikon), the inventor of scanning tunnelling microscopy (STM), described how the sizes of electronic devices to store and process information have decreased remorselessly for 40 years. By the year 2010 we should expect only $10^{3}$ atoms will be required to store 1 bit of information as compared to $10^{9}$ today. We are also moving towards two convergence points. The first is between supramolecular chemistry and solid state physics in constructing functional macromolecular devices. This was demonstrated by $\mathrm{H}$. Nejoh (Aono Atomcraft Project, Japan) in his presentation of a single electron transistor based on a STM-excited liquid crystal particle. However, we remain some way off as nature exploits a volume of $10^{6} \mu^{3}$ for biochemical switching (Na channel) whereas the latest HEMT devices are $10^{-1} \mu^{3}$ in volume, albeit much faster ( $10 \mu \mathrm{s}$ versus 2-3 ms: A.N. Broers, Cambridge). The second convergence is between real experiments carried out using relatively large number of atoms and computer experiments involving only a few. Computers will be increasingly able to predict the properties of aggregates that can be probed experimentally using $\mathrm{nm}$-scale techniques, especially since these techniques have the great advantage of allowing a clear choice of the working and control interactions.

So wherever small and high size, speed is needed one will inevitably see the assembly of functional $\mathrm{nm}$-scale units involving interdisciplinary and more complex manufacturing skills. For while progress in minaturisation has hitherto largely applied improved technology to well-developed scientific principles, the challenging future will exploit entirely new scientific concepts and phenomena.

\section{Imaging and Measurement}

\section{Local probe techniques}

\section{Investigations}

H.J. Güntherodt (Basel University) reviewed industrial applications of atomic force microscopy (AFM) and it derivatives for simultaneous force measurement and spectroscopy - important tools as they can be used for both conducting specimens and for industrially significant non-conducting materials. A range of properties can be examined and AFM had led to technological progress, important new results, intriguing exploratory work, and fundamental insights into new materials.

Several participants then detailed applications of nm-scale local probe methods for imaging and measurement. For semiconductors, H. Cerva (Siemens, Munich) described how an AC-STM method gives high resolution and high sensitivity measurements of the distance between source and drain regions in pn junctions, anticipating that it would one day complement conventional methods. H. Salemink (IBM Rüschlikon) examined in greater detail and precision the structural and electronic aspects of another type of semiconductor structure, namely epitaxial multilayers grown by MBE and MOVPE. Ultra-high vacuum STM was used with transversely cleaved cross-sections and a Wheatstone bridge circuit between the tip and the epilayer substrate to examine terracing, local ordering and the presence of additional phases, and defects in lattice-matched alloys: electronic aspects include valance band and bandgap levels at the nm-scale, fluctuations of the electronic homogeneity due to alloy variations, grown-in impurities, and local effects of doping. The interface can only be defined to 1-2 monolayers in GaAs/AIGaAs epilayers and $i-V$ spectroscopy shows an electronic transition region of 5 unit cells and two features arising from surface states. Oxygen impurities are associated with larger terrace steps of 8 unit cells. The AIGaAs ternary layers reveal atomic-scale composition fluctuations (bright regions in the cover illustration) with the Al favouring extended areas: $O$ and $C$ is located preferentially in this phase, and $\mathrm{Ga}$ atoms are not clearly defined at the epilayer interfaces, thus accounting for the interfacial charge density transition.

The majority of the presentations describing applications of local probe techniques where more quantitative. W. Hanrieder (Siemens, Munich) showed that STM had been used to identify conically shaped nucleation sites for nucleate boiling on thin tantalum films used to heat ink in ink-jet printers. T. Hellmuth (Carl-Zeiss, Oberkochen) discussed applications of STM for evaluating optical components (solvent residues on laser mirrors, the sharpness and regularity of gratings, etc.). The stich-probe technique was adopted to avoid frictional forces, the newer and faster non-contact mode using an oscillating tip being considered unreliable owing to the formation of bubbles in some experiments. For ceramic glasses, F. Creuzet (CNRS/St. Gobain, Aubervilliers) argued that the ultimate goal of $\mathrm{nm}$-methods is to resolve atomic structure and site chemistry. Meanwhile, they are being used to examine material science problems at the $>1 \mathrm{~nm}$ scale and for routine materials characterization at the $>10 \mathrm{~nm}$ scale.

H. Fuchs (BASF, Ludwigshafen) focussed on applications of STM to polymers, noting that materials of commercial significance often rely upon sub-micron structures. Some results were interesting such as the observation of a 3-5 times smaller helical pitch for a stretched polymer film deposited on graphite. AFM has recently been used to investigated pinholes in polymeric films and for materials development. The conclusion - AFM and STM are complementary except that AFM offers greater opportunities in materials development. The initial goal had been to examine defect structures in films grown on ordered surfaces (graphite, $W \mathrm{We}_{2}$ ). Molecular modelling is now being used to explain certain features observed during the growth of metallic films by electrodeposition and evaporation (regular trimeric arrays on $\mathrm{WSe}_{2}$ - see cover) - observations having implications for the production of $\mathrm{nm}$-scale structures.

\section{Techniques}

Applying STM and AFM to electrochemistry has required the development of special techniques $(\mathrm{H}$. Siegenthaler, Bern University). In AFM under potentiostatically controlled deposition the probe is not an electrode; in STM, charge transfer can be stimulated in scanning electrochemical microscopy where reactants are simultaneously oxidized and reduced in the STM gap. Three types of potentiostatic circuits are employed and several cell designs have been evaluated; probe tips invariably need coating (e.g., with $\mathrm{W}, \mathrm{Pt}, \mathrm{Au}$ ) and there remains vast scope for exploiting different electrodes. Most applications to-date involve simple imaging but there are fundamental issues which need tackling, including local probing of the electrolyte/electrode interface to measure barrier heights. Dr. Siegenthaler reported some results for metallic electrodes showing interesting morphological features, site-selective kinetics, time-dependent phenomena, and ordering. Recent work with ionic membranes polymerising on amorphous carbon has revealed areas with a $1.5-2.0 \mathrm{~nm}$ periodicity: the effect of ion additions on this unexplained structure is being examined as it may be useful for nm-scale manufacturing. All-in-all one can say atomic probe methods in electrochemistry are "out of their infancy".

R. Bendt (IBM, Rüschlikon) described enhanced photon emission techniques where light generated in and around the tunnelling gap is collected and analyzed with a grating spectrometer. Two regimes can be identified (tunnelling and field emission) where different resonance processes give different optical spectra. Tunnelling spectra can be interpreted in terms of inelastic excitation of radiatively coupled tipsurface plasmon modes resulting in enhancement of the signal intensity allowing both spectroscopy and mapping: different features such as surface steps, heterostructure layers and possibly dislocations show contrast (a spectacular example is a nmscale bump on a Ag surface glowing like a "light bulb"). Modelling the tip as a metallic sphere in a high vacuum yields calculated spectra in agreement with experimental data for clean $\mathrm{Ag}$ surfaces. Surprisingly, sufficient photon intensity is observed for transition elements so there are some opportu- 
nities for chemical mapping (e.g. titanium oxide). For semiconductors such as CdS, spectra agree with conventional electroluminescence spectra measured using trans mission electron microscopy (TEM); comparisons are employed to investigate elastic and inelastic tunnelling paths. Lasing action had been looked for and it is difficult to perform useful experiments in air.

H. Strecker (IBM, Mainz) described the use of local probe methods to evaluate mag netic storage discs where circumferential groves are ground to prevent the read/write head sticking to the polished and coated NiP layer. Magnetic force microscopy where the resonance frequency of the tip changes on interacting with the sample gives a complex force profile on moving along the $6.5 \mu \mathrm{m}$ long magnetic domains. A semi-automated STM instrument has also been designed for measuring surface profiles. A major problem was reproducibility of the STM tip, an issue addressed by $M$. Steadmen (NPL, UK) in describing applications of local probe methods in metrology. Nanometre-scale metrology falls on a unique area in amplitude-wavelength space, where the ability to measure surface features of a given wavelength and amplitude is defined by well-established geometric constraints such as the tip radius and taper (provided urgently needed $\mathrm{nm}$-scale standards are available). 0 . Woltjer (Institut für Mikrostrukturtechnologie, Wetzlar) extended the discussion in describing non-contact AFM where the tip is vibrated under constant force gradient control at $1-10 \mathrm{~nm}$ from the surface. The main advantage is no restriction regarding the tip radius and angle thus allowing deep features to be measured without sticking. Conventional (contact) AFM gives atomic resolution so is more important for scientific rather than routine investigations. He described how probes are fabricated, with the more durable silicon nitride types with small mechanical $Q$-factors preferred for contact AFM in spite of their large tip radius and angle $(500 \mathrm{~nm}$, $\left.70^{\circ}\right)$ : softer Si types having a small tip size and radius $\left(<100 \mathrm{~nm}, 30-60^{\circ}\right)$ are preferred for non-contact AFM and other microscopies.

V.T. Binh (University, Villeurbanne) considered in detail the fabrication of tailored tips for meeting more exacting requirements such as a single atom capable of acting as a coherent source of ions or electrons. $\mathrm{Cal}$ culations show that by supporting a $2 \mathrm{~nm}$ radius protrusion on a $50 \mathrm{~nm}$ radius tip one should be able to localize the electrostatic field at three atoms or one atom on the ("teton") STM tip. Manufacturing these tips calls upon established principles for creating $\mathrm{nm}$-scale structures, specifically, surface diffusion and roughening effects arising on scanning the tip temperature.

\section{Non-local probe techniques}

The participants were reminded of the rich diversity of non-local probe techniques for imaging and measurement. G.W. Smith (Oxford) described how field ion microscopy (FIM) has developed chemical analy- sis techniques based on passing a restricted part of the ion beam through a detector to give a "bore hole" analysis through the FIM specimen tip. The latest development is full, computer-based, reconstruction of the atomic chemical composition in three-dimensions using a channel plate and a positionsensing anode structure. Atoms removed from successive rings are mapped to give remarkably instructive isosurfaces of constant composition. The example on the cover shows the spinodally decomposed, continuous interpenetrating surface for $\mathrm{Cr}$ with a characteristic scale of $\approx 15 \mathrm{~nm}$ observed in an irradiated stainless steel.

Transmission electron microscopy (TEM) is also a powerful tool for investigating $\mathrm{nm}$ scale structures (W. Stobbs, Cambridge). Unlike STM, TEM effectively applies a fast Fourier transform with parallel data acquisition so atom positions and chemistry are convoluted. The trend now is towards image filtering but in the meantime one must use conventional methods that are relatively insensitive to elastic scattering. One takes a through-focus series of images of edge-on structures (e.g. grain boundaries, multilayers) and examines changes in the Fresnel fringes. Dr. Stobbs defined limits to what could be achieved in practice, noting that it will be difficult to obtain three dimensional information. R. Hauert (EMPA Dübendorf) in outlining applications of depth profiling techniques based upon the successive removal of layers with Auger or ESCA analysis remarked that $80 \%$ of work involved oxidation or contamination.

Analyses of the mechanical properties of few-atom structures by J.B. Pethica (Oxford) represent one the most innovative applications of classical methodologies to the nanometre regime. Consider first the mechanical response at or near the theore tical lattice strain: in a simple hardness test the measured hardness increases rapidly and approaches the theoretical lattice strength as the indent depth decreases below about $10 \mathrm{~nm}$, where dislocations become unimportant. Curious features are also observed on microloading stress-strain curves owing to the onset of new pheno mena. The standard scratch test has been modified to impose a sub-angstrom level $100 \mathrm{~Hz}$ AC loading component on a DC component during indentor displacement and has been used to measure micro-creep by suddenly changing the stress level.

\section{Manufacture and Manipulation}

\section{Local methods}

Nanometre-scale devices are being produced for non-electronic applications so fabrication techniques for $\mathrm{nm}$-scale struc tures no longer merely represent academic curiosities. C. Quate (Stanford), in review ing probe-type techniques, highlighted STM enhanced diffusion effects, decomposition, material transfer, and under-surface modification. Regarding mechanical approaches: molecular dynamic simulations (J.B. Pethica) of the interaction of a sharp $W$ tip with lead indicated that the dominant inelastic atom displacement is diffusive; disloca- tions and other defect structures appear to be less stable than liquid-like structures, showing the importance of the elastic far field even for inelastic flow.

Exploratory work on the stability of $\mathrm{nm}$ scale structures - an essential feature in eventual applications - was reported. For instance, atomic order remains undisturbed and stable for $3 \mu \mathrm{m}$ wide STM indents in $\mathrm{WSe}_{2}$ (H. Fuchs). G. Walmsley (Belfast) has examined what happens to pits resulting from STM tip "crashes" and estimates that their disappearance corresponds to a surface diffusion coefficient $D_{\mathrm{s}}=10^{-18} \mathrm{~cm}^{2} / \mathrm{s}$. Deposited features, on the other hand, are very much more stable, perhaps because $D_{\mathrm{s}}$ around the bottom of a protrusion is reduced owing to a higher coordination number for a surface atom.

\section{Nanolithography}

A.N. Broers (Cambridge) argued that conventional integrated circuits with dimensions above $0.1 \mu \mathrm{m}$ will meet most needs until perhaps the year 2010. Smaller dimensions are being used to determine device physics, limits to transistor performance and whether quantum effects can be harnessed. Developments in optical lithography (notably lenses and surface-acting resists) render the method satisfactory down to $0.2 \mu \mathrm{m}$. X-ray lithography, which has some potential for further reductions, is in its present form incapable of producing sub-0.1 $\mu \mathrm{m}$ dimensions (using soft $\mathrm{X}$-rays with thick resists and thin masks to suppress fringes) owing to focussing errors on rippled wafers. Scanning may extend the range below $0.1 \mu \mathrm{m}$.

To date, all useful nanostructure devices have been produced with electron beam lithography in conventional resists $15 \mathrm{~nm}$ tracks and $0.16 \mu \mathrm{m}$ diameter rings useful for novel types of discrete electronic devices) but it is difficult to imagine large scale applications as writing a pattern takes too long. Moreover, the resolution of electro-optic systems is not replicated in the device fabrication process (a $1 \mathrm{~nm}$ beam writes a $10 \mathrm{~nm}$ line) owing to line broadening by the excitation of secondary electrons in the resist.

Hole drilling by direct sublimation, e.g., of $\mathrm{NaCl}$, gave, in 1976, $2 \mathrm{~nm}$ dots on $4 \mathrm{~nm}$ $(0.8 \mathrm{~nm}$ holes have been achieved but not in useful substrates); working devices based on arrays of holes have not yet been produced. A new process based on the direct nano-patterning of silica - a standard component of transistors - has been developed based on work in the 1960's at Westinghouse. Exposure to an electron beam enhances the etch rate in buffered HF acid and parallel $10 \mathrm{~nm}$ lines with $15 \mathrm{~nm}$ centers (three times less than for conventional PMMA resists - see cover illustration) have been produced in both single crystal and polycrystalline silicon. As lithography continues to develop, novel atom-scale approaches should not be overlooked, such as the manufacture of periodic structures exploiting chemical reactions at step ledges on the surfaces of highly misoriented metals.

P.G. Boswell 